\title{
IL CONCETTO DI LEGITTIMITÀ: FRA TEORIA E RICERCA EMPIRICA
}

di Pamela Pansardi

Il concetto di legittimità è senza dubbio uno dei più diffusi ed ambigui del linguaggio politico. L'attribuzione di legittimità ad un certo oggetto politico (il potere politico, un'istituzione politica, un dato sistema politico) sembra consistere in un'attribuzione di valore positivo a quello stesso oggetto. Tuttavia, all'interno della letteratura sulla legittimità, non sembra esservi accordo su quale proprietà (o insieme di proprietà) un certo oggetto debba presentare per essere considerato legittimo.

Scopo preliminare di questo lavoro sarà quello di presentare una breve panoramica della letteratura sulla legittimità, sottolineandone differenze e similarità. Si cercherà, quindi, di interpretare il contributo delle varie posizioni ai fini della formulazione di una definizione empirica del concetto di legittimità, nonché della definizione dei canoni di una possibile operazionalizzazione di tale concetto, che sia valida e utilizzabile a fini comparativi. In questo saggio, dopo un'introduzione all'analisi formale del concetto di legittimità, verranno passati in rassegna alcuni contributi contemporanei alla definizione e alla misurazione empirica della legittimità di un sistema politico. Verranno qui distinti gli approcci 'macro', così definiti poiché intesi ad investigare la legittimità di un sistema politico a partire dall'analisi della sue caratteristiche istituzionali, e gli approcci cosiddetti 'micro' 1 , quelli ovvero volti ad investigare la legittimità di un sistema politico a partire dallo studio dell'opinione dei membri stessi della comunità politica a

Dipartimento di Scienze Politiche e Sociali, Università di Pavia.

1 M.S. Weatherford, Measuring Political Legitimacy, in "American Political Science Review", n. 1, 1992, p. 149. 
cui il sistema fa riferimento. Nella parte conclusiva, verrà inoltre presentato il tentativo di operazionalizzazione del concetto di legittimità proposto da Bruce Gilley², che, inserendosi nell'ambito degli studi a livello micro, sembra in grado di offrire una interessante soluzione ad alcuni dei limiti metodologici degli stessi.

\section{L'analisi del concetto di legittimità}

Le varie definizioni di legittimità presenti in letteratura possono essere spiegate facendo riferimento ai diversi termini inclusi nel seguente enunciato:

$A$ è legittimo (1) per $B$ sulla base di $C$

Per evidenziare, quindi, i principali punti di disaccordo delle varie definizioni di legittimità, sembra possibile fare riferimento alle varie interpretazioni che esse offrono delle tre variabili sopra riportate ${ }^{3} . A$ rappresenta l'oggetto a cui viene attribuita la proprietà ' 1 '. Risponde alla domanda: 'legittimità di che cosa?'. $B$ rappresenta il soggetto della legittimità, indica ovvero l'agente che valuta se $A$ possiede la proprietà ' 1 '. Risponde alla domanda: 'chi decide della legittimità?'. $C$ rappresenta il criterio che definisce l'attribuzione di ' 1 ' ad $A$. Risponde alla domanda: 'in base a che cosa un oggetto può dirsi legittimo?'.

L'analisi delle varie interpretazioni delle tre variabili sopra riportate consente, quindi, di comprendere più a fondo le somiglianze e le differenze fra i vari contributi teorici allo studio della legittimità. Al fine, quindi, di introdurre l'esposizione analitica di alcune delle varie concezioni di legittimità, è ora utile proporre, in chiave schematica, i significati che possono essere attribuiti alle tre diverse variabili.

2 B. GILLEY, The Meaning and Measure of State Legitimacy: Results for 72 countries, in "European Journal of Political Research", n. 3, 2006, pp. 499-525; B. GiLlEY, The Determinants of State Legitimacy: Results for 72 Countries, in "International Political Science Review", n. 1, 2006, pp. 47-71.

3 R.D. LAMB, Measuring Legitimacy in Weak States, CISSM Working paper, Georgetown University, 2005, p. 6. 
A. L'oggetto della legittimità. Nell'ambito dello studio della politica, il concetto di legittimità è generalmente riferito ad una certa interpretazione o manifestazione del potere politico. L'attribuzione di legittimità può, quindi, essere indirizzata a diversi tipi di oggetti, fra i quali:

1. una certa politica pubblica;

2. uno stato, inteso come sistema di organizzazione territoriale che presiede ad una comunità politica;

3. il ruolo, o le azioni di uno stato nell'arena politica internazionale;

4. la struttura socio-economica, ovvero il sistema di distribuzione delle risorse sociali ed economiche fra i cittadini;

5. un governo, ovvero gli occupanti attuali delle cariche politiche;

6. un sistema politico (o regime), ovvero l'insieme delle istituzioni politiche e amministrative, delle regole che presiedono al loro funzionamento, e dei valori che li caratterizzano ${ }^{4}$.

Delle varie interpretazioni dell'oggetto del concetto politico di legittimità, in questo studio si privilegerà l'ultima. Ci si concentrerà, quindi, esclusivamente su quei contributi che studiano la legittimità come proprietà di un sistema politico. Le variabili $B$ e $C$, analizzate qui di seguito, costituiranno i criteri fondamentali per la distinzione fra $\mathrm{i}$ diversi tipi di approcci.

B. Il soggetto della legittimità. Per soggetto della legittimità possiamo intendere l'agente (collettivo o individuale) che è chiamato a decidere dell'attribuzione della legittimità ad un certo oggetto. All'interno della letteratura sulla legittimità politica, vengono presi in considerazione due tipi di agenti:

1. un osservatore esterno (ad esempio, lo scienziato politico, il filosofo politico, l'opinione pubblica internazionale, ecc.);

2. il soggetto stesso del potere politico, ovvero l'insieme dei membri della comunità politica a cui un sistema politico fa riferimento.

C. Il criterio di legittimità. L'ultima variabile è riferita al criterio sulla base del quale la legittimità viene attribuita ad un sistema politico. I criteri che presiedono la definizione di legittimità possono essere di due tipi:

1. criteri oggettivisti: cosa è legittimo è definibile sulla base di criteri

4 M. Stoppino, Potere e teoria politica, Milano, Giuffrè, 2001, p. 327. 
universalmente validi. La definizione di 'legittimo' sopravviene all'accordo circa la definizione di regime politico 'giusto'. La legittimità è una proprietà intrinseca del suo oggetto;

2. criteri soggettivisti: cosa è legittimo è relativo all'opinione degli individui membri di un dato sistema politico. Non esiste una concezione sostantiva di 'sistema politico legittimo' che sia universalmente valida ${ }^{5}$. La legittimità è una proprietà della relazione fra oggetto e soggetto.

E' importante notare come le varie concezioni di legittimità presentino una certa correlazione fra le due diverse interpretazioni di $B$ e $C$, ovvero, fra i due tipi di criteri di legittimità e le due interpretazioni del soggetto. Definizioni oggettiviste di legittimità, in genere, sono legate ad una valutazione del rapporto di potere in capo ad un osservatore esterno. Definizioni soggettiviste, al contrario, concepiscono l'attribuzione della legittimità in capo a coloro che vi sono sottoposti: in questo senso, il criterio di legittimità non può dirsi unico e condivisibile, bensì relativo allo specifico contesto. Definizioni di tipo oggettivista si ritrovano generalmente in ambito di filosofia politica. I criteri di definizione della legittimità sono, in questo caso, criteri normativi. L'esempio classico di definizione soggettivista di legittimità è, invece, quello offerto da Max Weber 6 , per il quale la legittimità consiste nella credenza della legittimità di un potere da parte di coloro che vi sono sottoposti. Un tipo 'misto' di definizione di legittimità è, infine, quello proposto da David Beetham in The Legitimation of Power?. Se per Beetham la legittimità di un sistema politico è definibile tramite criteri oggettivi (la conformità con regole prestabilite; la giustificabilità delle regole in base a credenze condivise; la manifestazione di consenso da parte dei subordinati $)^{8}$ e quindi verificabile da un osservatore esterno, il contenuto di questi criteri è soggettivo, varia, ovvero, in base allo specifico contesto di riferimento. In questo senso, la legittimità non è intesa come

5 R.D. LamB, Measuring Legitimacy in Weak States, cit., p. 9.

6 M. WeBer, The Theory of Social and Economic Organization, a cura di T. PARSONS, New York, Free Press, 1964, p. 382.

7 D. Beetham, The Legitimation of Power, London, MacMillan, 1991. Si veda, inoltre, C. LoRD, D. BEetham, Legitimatizing the UE: Is There a 'Post-parliamentary Basis' for its Legitimation?, in "Journal of Common Market Studies", n. 3, 2001, pp. 443-462.

8 D. Beetham, The Legitimation of Power, cit., p. 19. 
qualità di un particolare 'tipo' di regime politico - non è definita tramite 'valori' universalmente validi - ma viene valutata in ogni singolo contesto in base al soddisfacimento delle tre condizioni indicate.

Lo schema preliminare formulato nelle pagine precedenti sarà utile ai fini della presentazione dei vari contributi teorici allo studio della legittimità, non solo perché ne ha anticipato alcuni temi, ma perché fornirà gli strumenti di analisi per meglio comprendere e distinguere le varie posizioni.

\section{Gli studi sulla legittimità}

La letteratura recente sulla legittimità ha distinto due livelli di analisi della legittimità di un sistema politico: il livello macro e il livello micro9. Il livello macro concerne l'analisi dell'organizzazione stessa del potere politico, delle sue 'caratteristiche formali'; il livello micro, invece, è riferito alle attitudini dei soggetti del potere verso di esso. La distinzione fra i due livelli presiede alla divisione principale fra i due principali filoni empirici di studio della legittimità. Studi a livello macro si sono concentrati sull'analisi della legittimità di particolari sistemi politici sulla base di criteri condivisi, studiandone l'organizzazione, il funzionamento e la performance istituzionale tramite la metodologia di analisi delle istituzioni politiche tipica della politica comparata e della scienza politica in generale. Studi a livello micro, invece, si sono occupati di valutare la percezione, da parte dei soggetti del potere, della fiducia nelle istituzioni che li governano e in coloro che occupano le cariche pubbliche, nonché della bontà delle politiche pubbliche prodotte. Metodo d'indagine privilegiato è l'opinion survey.

\subsection{Lo studio della legittimità a livello macro}

A livello macro, come è facile intuire, l'investigazione della legittimità di un sistema politico si basa su una definizione del concetto stesso di legittimità in chiave oggettivista. Secondo questa prospettiva

9 M.S. Weatherford, Measuring Political Legitimacy, cit., p. 149. 
è possibile definire univocamente $\mathrm{i}$ criteri che presiedono la definizione di un sistema politico legittimo. Un sistema di organizzazione del potere politico è, quindi, considerato legittimo solo se soddisfa un certo numero di criteri, identificabili razionalmente e condivisibili: "Il concetto [di legittimità] dovrebbe significare la valutazione normativa di un regime politico: la correttezza delle sue procedure, la giustificazione delle sue decisioni, e l'equità con cui tratta i soggetti del potere $10 "$.

E' da notare che analisi di questo tipo generalmente pongono alla propria base l'ideale democratico quale condizione di legittimità: se la legittimità è da considerarsi quale proprietà di un governo 'giusto' (o migliore), e il governo 'giusto' è un governo costruito sugli ideali di democrazia, allora la legittimità dipenderà dal soddisfacimento, da parte di quel governo, dei criteri di democraticità. Dal punto di vista dell'analisi empirica, l'approccio macro è molto diffuso nella letteratura circa la legittimità dell'Unione Europea: in quest'ultimo caso, la sovrapposizione dei due criteri (legittimità e democraticità) è talmente forte da spingere, in alcuni casi, ad assumere una relazione biunivoca fra deficit democratico e deficit di legittimità. L'approccio macro allo studio della legittimità è quindi ispirato da criteri universalistici. 'Il diritto dei governanti a essere obbediti' è quindi funzione di una valutazione razionale dell'organizzazione stessa del potere politico. $\mathrm{Su}$ ispirazione della massima pronunciata da Lincoln nel suo discorso di Gettysburg del 1863, la legittimità è stata spesso definita come la proprietà di un governo 'del popolo, tramite il popolo, e per il popolo'. Le varie componenti della legittimità sono di conseguenza state definite seguendo questa tripartizione. 'Governo del popolo' implica partecipazione politica. 'Governo tramite il popolo' sottolinea il ruolo della rappresentanza politica. 'Governo per il popolo' indica, infine, l'efficacia politica. Studiare la legittimità di un sistema è, quindi, verificare empiricamente la misura in cui esso soddisfa tali criteri.

All'interno degli studi sull'Unione Europea, le suddette componenti della legittimità sono state riformulate alla luce di una teoria sistemica della politica ponendo specifica attenzione ai due lati del processo poli-

10 R. Grafstein, The Failure of Weber's Conception of Legitimacy: Its Causes and Implications, in "Journal of Politics", n. 2, 1981, p. 456. 
tico: quello dell'input quello dell'output ${ }^{11}$. Dal lato dell'input, quello che conta al fine della legittimità è che le preferenze dei governati abbiano un effettivo ruolo nella presa di decisione pubblica, che il governo sia, effettivamente un 'governo del popolo'. In questo senso, le dimensioni da investigare riguardano la partecipazione, la rappresentanza politica e il consenso. Dal lato dell'output, invece, si considera l'aderenza delle decisioni politiche alle preferenze formulate dai governati, ovvero l'efficacia del governo nel rispondere alle domande che riceve: il governo deve essere un 'governo per il popolo' ${ }^{12}$. Le dimensioni da investigare sono quindi collegate sia alla vera e propria performance del governo, che ai concetti di responsiveness e accountability dei rappresentanti13. Più in generale, i criteri che presiedono la formulazione di una concezione della legittimità a livello macro sembrano includere sia una prospettiva 'procedurale' che una 'consequenzialista': la legittimità del potere politico non è funzione esclusiva della sua organizzazione né della sua performance. Così come è illegittimo un sistema autoritario paternalista, che prende decisioni politiche che favoriscono gli interessi dei governati, è altrettanto illegittimo un sistema politico organizzato in maniera democratica che non riesce a garantire delle risposte efficaci alle domande dei cittadini. La dimensione procedurale (la presenza, ovvero, di determinate qualità del sistema politico dal lato dell'input, ad es. la bontà del meccanismo di rappresentanza) non è abbastanza per garantire la legittimità: l'incapacità, dal lato dell'output, di garantire il rispetto degli interessi dei cittadini deve essere in egual modo considerata ${ }^{14}$.

È da notare, tuttavia, come Scharpf, a questo riguardo, assuma una posizione leggermente diversa, affrontando il caso specifico dell'Unione Europea. Quello che conta, nel caso dell'Unione Europea, è che vi sia legittimità 'orientata all'ouptut'. Ciò, apparentemente, vedrebbe una riduzione del peso del ruolo attivo dei 'governati' (dal lato dell'input) nella formulazione di un criterio di legittimità: quello che

${ }^{11} \mathrm{~F}$. SCHARPF, Governare l'Europa. Legittimità democratica ed efficacia delle politiche nell'Unione Europea, Bologna, il Mulino, 1999.

${ }^{12} \mathrm{~F}$. SCHARPF, Governare l'Europa. Legittimità democratica ed efficacia delle politiche nell'Unione Europea, cit., p. 13.

${ }^{13} \mathrm{~F}$. SCHARPF, Governare l'Europa. Legittimità democratica ed efficacia delle politiche nell'Unione Europea, cit., p. 28.

${ }^{14}$ B. RothsteIn, Creating Political Legitimacy. Electoral Democracy Versus Quality of Government, in “American Behavioral Scientist”, n. 3, 2009, pp. 311-331. 
conta non è il rispetto della volontà dei cittadini, espresso tramite la manifestazione di preferenze, quanto l'efficacia della decisione politica ed il rispetto dei loro interessi. Scharpf sembrerebbe, quindi, a prima vista abbracciare un criterio puramente consequenzialista di legittimità. Tuttavia, Scharpf chiarisce il punto specificando che: 1) la legittimità input-oriented include necessariamente una visione del popolo come collettività dotata di una identità comune, condizione che il sistema politico europeo non soddisfa; 2) la legittimità output-oriented include essa stessa criteri di responsiveness e accountability, che - a differenza di quello che accade dal lato dell'input - non registrano la 'volontà del popolo', bensì definiscono un 'ambito di interessi comuni' 15 .

Sempre nell'ambito degli studi sulla legittimità dell'Unione Europea, un contributo recente è quello offerto da Vivien Schmidt ${ }^{16}$. Secondo Schmidt, nella valutazione della legittimità dell'Unione Europea, i criteri input e output-oriented non sono sufficienti: un terzo criterio deve essere integrato, quello che chiama di throughput-legitimacy, ed è riferito a quello che succede all'interno del processo decisionale dell'Unione Europea. Per Schmidt la throughput-legitimacy è definita sulla base de "l'efficacia, l'accountability, la trasparenza e l'inclusività del processo di governo17" e va investigata a partire dello studio delle "interazioni fra gli attori impegnati nel processo decisionale a livello Europeo ${ }^{18 "}$ ".

Uno schema più generale di quelle che sembrano essere le varie dimensioni della legittimità presenti negli studi a livello macro è offerto da Weatherford ${ }^{19}$ :

1. accountability: esiste un sistema di regole che garantisca un'ampia ed effettiva partecipazione, e che renda i governanti responsabili nei confronti dei governati?

2. efficienza: il governo è strutturato in modo da perseguire i fini della società evitando sprechi di tempo e risorse?

3. equità procedurale: l'organizzazione del governo è strutturata in

${ }^{15} \mathrm{~F}$. SCHARPF, Governare l'Europa. Legittimità democratica ed efficacia delle politiche nell'Unione Europea, cit., pp. 18-21.

16 V.A. SснміDт, Democracy and Legitimacy in the European Union Revisited: Input, Output and 'Throughput', in "Political Studies", n. 1, 2012, pp. 2-22.

17 V.A. ScHMIDT, Democracy and Legitimacy in the European Union Revisited, cit., p. 2.

18 V.A. SCHMIDT, Democracy and Legitimacy in the European Union Revisited, cit., p. 4.

${ }^{19}$ M.S. WeATHERFord, Measuring Political Legitimacy, cit.. 
modo da garantire che le questioni vengano risolte in modo regolare e prevedibile, e in modo che l'accesso all'arena decisionale sia aperto ed eguale per tutti?

4. equità distributiva: i costi e i vantaggi allocati dal sistema sono distribuiti equamente, o comunque giustificati?

Come è evidente, la definizione delle dimensioni da investigare al fine di valutare la legittimità di un sistema politico è, negli approcci macro, un'operazione normativa: un sistema politico può dirsi legittimo se e solo se soddisfa delle condizioni determinate in base a specifici criteri normativi. La legittimità, in questo senso, è investigata non come un fatto, bensì come un valore. Il tentativo di offrire una definizione empirica di legittimità all'interno dell'approccio macro - una definizione, ovvero, operazionalizzabile - non può, nella maggior parti dei casi, esimersi dal porre l'attenzione sulla sua stretta relazione con il concetto di democrazia. Poiché le analisi macro fanno riferimento agli stessi indicatori utilizzati per valutare la 'democraticità' o la 'qualità di una democrazia', la sovrapposizione concettuale è tale da rendere inutile la distinzione dei due concetti: Se la legittimità è valutata in relazione di identità con la bontà della democrazia, allora il concetto di legittimità è inutile ai fini della ricerca empirica, poiché non 'spiega' nulla di più di quello già spiegato con il concetto di 'democraticità'.

\subsection{Lo studio della legittimità a livello micro}

Gli studi della legittimità cosiddetti 'a livello micro', come già accennato, non si focalizzano sull'analisi dei requisiti formali del sistema politico, bensì investigano la legittimità del sistema a partire dalle opinioni e credenze dei cittadini. La legittimità, in questo caso, non è considerata quale una proprietà intrinseca di un sistema politico; è definita in termini soggettivistici, sulla base del rapporto fra il sistema politico e la comunità di riferimento. Lo studio della legittimità a livello micro prende quindi come punto di partenza la classica concezione weberiana di legittimità, che individua nelle 'credenze' dei soggetti al potere il locus stesso della legittimità del regime ${ }^{20}$. Un potere, nella concezione webe-

${ }^{20}$ M. WeBer, The Theory of Social and Economic Organization, cit., p. 382. 
riana, è legittimo se e solo se coloro che vi sono sottoposti lo reputano tale. La posizione di Weber, è, come è evidente, una posizione soggettivista: non esiste un unico criterio oggettivo sulla base del quale un sistema politico possa dirsi legittimo o meno. Gli unici criteri di legittimità sono quelli utilizzati dai governati per giudicarlo. Distinguendo fra i tre ideal-tipi di legittimità - carismatico, tradizionale, e legale-razionale -, lo scopo di Weber è spiegare (e non valutare) la base motivazionale per cui diverse comunità politiche hanno conferito o conferiscono ai propri governanti 'il diritto di essere obbediti'. La legittimità, secondo Weber, non è da considerarsi una proprietà che possa essere attribuita ad un sistema politico in base alla sua stessa 'natura' (non è da ricercare nella 'forma' del potere, $o$ in caratteristiche specifiche della sua organizzazione). E' piuttosto definita dal rapporto stesso che lega i governanti e i governati: è una credenza, non un fatto, del potere.

E' da notare come la stessa nozione di 'credenza' sia stata causa di alcune ambiguità nell'interpretazione della concezione weberiana di legittimità. Alcuni autori, infatti, hanno interpretato il rapporto fra il potere politico e la 'credenza nella legittimità' in un senso più esteso. Per Weber, la 'credenza della legittimità' è la base, o fonte, del potere politico. Alcuni hanno invece suggerito che la legittimità sia da considerarsi sia una fonte, sia, e soprattutto, un effetto (più o meno intenzionale) del potere politico. La credenza nella legittimità è stata, ovvero, vista come un qualcosa che il potere politico tende a creare e riprodurre. Da un lato è stata spiegata come frutto di azioni intenzionali di coloro che detengono il potere al fine di garantire l'acquiescenza di coloro che vi sono sottoposti. Dall'altro, la credenza nella legittimità è stata spiegata come una caratteristica sistemica: la sua riproduzione non è il frutto di azioni individuali, bensì l'esito del normale funzionamento del sistema che tende a riprodurre se stesso. Lipset scrive: "La legittimità concerne la capacità del sistema di generare e mantenere la credenza che le istituzioni politiche vigenti siano le più appropriate per la nostra società21". Contro questa interpretazione della nozione di legittimità sembra funzionare l'obiezione di Beetham ${ }^{22}$,

${ }^{21}$ S.M. LIPSET, Political Man: The Social Bases of Politics, New York, Anchor Books, 1963, p. 65. Si veda, inoltre, S.M. LIPSET, Some Social Requisites of Democracy: Economic Development and Political Legitimacy, in "American Political Science Review", n. 1, 1959, pp. 69-105.

22 D. Beetham, The Legitimation of Power, cit., p. 13. 
secondo la quale, se così fosse, le crisi di legittimità, e quindi, l'instabilità politica, potrebbero essere totalmente spiegate in termini di incapacità del sistema di 'far credere' di essere legittimo, piuttosto che dovute ad un cambiamento, indipendente dal sistema stesso (e quindi causato, ad esempio, dall'ingerenza di fattori esterni), nei valori e nelle credenze dei governati.

Ai fini della ricerca empirica, una efficace descrizione del rapporto fra il sistema politico e la sua attribuzione di legittimità sembra essere quella che emerge dalla definizione di Easton di sostegno diffuso: "una 'riserva' di atteggiamenti [...] favorevoli che spingono i membri ad accettare o tollerare outputs a cui si opporrebbero, o i cui effetti potrebbero essere dannosi per la realizzazione dei loro desideri23". È da notare che Easton considera il sostegno diffuso al sistema politico e la sua legittimità come due concetti distinti. Secondo Easton, la legittimità costituisce una delle componenti del sostegno politico, insieme alla fiducia nel sistema politico ${ }^{24}$, ed è definita come la convinzione che sia: "giusto ed appropriato [...] accettare ed obbedire all'autorità e conformarsi alle sue richieste. [La legittimità] riflette il fatto che in modo più o meno esplicito [un individuo] vede questi oggetti come conformi ai suoi principi morali, al suo senso di cosa sia giusto e appropriato nella sfera politica ${ }^{25}$ ". La legittimità sembra poter essere definita, quindi, come la proprietà di quel sistema politico nel quale gli atteggiamenti dei governati sono orientati favorevolmente all'accettazione di qualsiasi decisione prodotta, a prescindere dal contenuto della decisione stessa. Sebbene per Easton legittimità e sostegno diffuso siano da considerarsi analiticamente come due concetti distinti, dal punto di vista empirico, come è testimoniato dai numerosi studi in cui sostegno e legittimità sono utilizzati come sinonimi ${ }^{26}$, lo studio del primo non può che passare attraverso l'analisi del secondo.

Dal punto di vista empirico, gli studi a livello micro volti all'analisi

23 D. Easton, A Systems Analysis of Political Life, New York, Wiley, 1965, p. 273.

${ }^{24}$ D. EAston, A Re-Assessment of the Concept of Political Support, in "British Journal of Political Science", n. 4, 1975, p. 447.

25 D. EAston, A Systems Analysis of Political Life, cit., p. 278.

26 Per una discussione si veda H. Sсhмiтt, J. ThOMASSEn (a cura), Political Representation and Legitimacy in the European Union, Oxford, Oxford University Press, 1999. 
della legittimità dei sistemi politici contemporanei si sono concentrati sulla misurazione del sostegno di cui un certo sistema politico gode all'interno della comunità politica di riferimento. Metodologia principale è stata quindi l'analisi degli atteggiamenti dei cittadini nei confronti del sistema politico sulla base di opinion surveys. Un contributo classico in questo senso è lo studio curato da Pippa Norris in Critical Citizens $^{27}$. Scopo dello studio è un'analisi empirica del concetto di sostegno politico - al fine di corroborare la tesi di una 'crisi di sostegno delle democrazie'. Norris distingue cinque dimensioni del sostegno politico: 1) il sostegno alla comunità politica; 2) il sostegno agli attori politici; 3) il sostegno ai principi del regime; 4) il sostegno al funzionamento del regime; 5) il sostegno alle istituzioni. E' da notare come, mentre le prime due dimensioni ricalcano perfettamente due delle dimensioni della distinzione eastoniana degli oggetti di sostegno (la comunità politica e le autorità $)^{28}$, le ultime tre consistano in una specificazione della terza dimensione eastoniana detta del 'regime politico'. La necessità di distinguere fra tre diversi tipi di sostegno al regime è spiegata, da Norriss, in base alle diverse opinioni che i cittadini possono avere sull'una o sull'altro: pur condividendo i principi del regime, ad esempio, i cittadini possono essere critici sul suo funzionamento. Il livello di sostegno alle varie dimensioni viene quindi misurato sulla base di risposte a questionari (sulla base di preesistenti dataset). Gli indicatori del sostegno vengono quindi distinti sulla base delle cinque dimensioni:

1. il sostegno alla comunità politica è analizzato in termini di identificazione con la comunità politica o con la nazione. Indicatori rilevanti sono considerati i dati su risposte a domande del genere: 'quanto orgoglioso sei di essere inglese/americano/tedesco, etc.' e 'combatteresti per proteggere la tua nazione?';

2. il sostegno agli attori politici è misurato sulla base di rilevazioni generali della fiducia agli attori politici e di valutazioni della performance di attori specifici;

3. il sostegno ai principi del regime, invece, è ricercato sulla base di tre indicatori: la definizione di democrazia; l'accordo con l'idea

27 P. Norris, Critical Citizens: Global Support for Democratic Government, Oxford, Oxford University Press, 1999.

28 D. Easton, A Systems Analysis of Political Life, cit.. 
che la democrazia sia la forma di governo migliore; e la preferenza per la democrazia rispetto ad un regime autoritario;

4. il sostegno al governo è rilevato sulla base di risposte dirette a domande concernenti il livello di soddisfazione sul funzionamento della democrazia nel proprio paese e (in casi specifici) su comparazioni con il regime politico precedente;

5. il sostegno alle istituzioni, interpretato come sostegno alla struttura del regime - e non agli attuali occupanti delle cariche - è valutato, infine, sulla base di risposte a domande sul rispetto dell'autorità e sulla fiducia nei confronti di specifiche istituzioni.

$\leftarrow$ Se è indubbio che l'utilizzo di opinion surveys possa incorrere nel rischio di ottenere risultati fortemente influenzati dall'opinione degli intervistati sul governo in carica (anche nel caso in cui le domande siano indirizzate a verificare la valutazione della 'performance' e delle 'istituzioni' del 'regime', e non una valutazione degli attuali occupanti delle cariche) è possibile che l'utilizzo di serie temporali abbastanza lunghe (per i paesi dove i dati sono disponibili) permetta allo studio di Norris di offrire una risposta valida al problema della misurazione del sostegno. E' da notare che un simile modello di analisi empirica delle dimensioni della legittimità è stato utilizzato da Schmitt e Thomassen in Political Representation and Legitimacy in the European Union'29, dove tuttavia la dimensione 'sostegno agli attori politici' viene esclusa poiché, a parere degli autori: "un sostegno negativo alle autorità $[\ldots]$ può coesistere con la credenza nella legittimità del regime 30 ". Il problema qui sottolineato è rilevante ai fini di ricerca empirica: quanto deve incidere la misurazione delle opinioni sugli attuali governanti nella rilevazione della legittimità di un sistema politico? E', in linea con la posizione di Schmitt e Thomassen, più efficace, al fine di misurare le legittimità del sistema politico, escludere dagli indicatori la misura del sostegno all'attuale governo, al fine di evitare eccessive distorsioni sulla base di quest'ultima?

${ }^{29}$ H. Schmit, J. Thomassen (a cura), Political Representation and Legitimacy in the European Union, cit..

${ }^{30}$ H. Schmitt, J. Thomassen (a cura), Political Representation and Legitimacy in the European Union, cit., p. 13. 
$\leftarrow$ Il problema, come è stato precedentemente evidenziato, è probabilmente più ampio. L'opinione sul governo in carica, infatti, rischia di influenzare le risposte dei cittadini anche riguardo ai temi del funzionamento e alle strutture del regime. Più che registrare il sostegno al funzionamento del regime, quindi, si rischierebbe di registrare il sostegno specifico alla performance ${ }^{31}$, in termini di output, dell'attuale governo, e più che un sostegno all'organizzazione delle strutture del regime, si rischierebbe di registrare, di nuovo, un sostegno agli attuali occupanti delle cariche pubbliche. "Domande generali [...] riguardo la 'fiducia' nel governo o particolari istituzioni non sono necessariamente un passo verso [la misurazione] della legittimità del regime, poiché gli intervistati tendono a comprendere tali domande come 'fiducia verso particolari occupanti delle cariche' e verso quello che fanno ${ }^{32}$ ". Questo è, indubbiamente, un problema di metodologia della ricerca sociale. Se la dimensione dei dati lo consente, come nello studio proposto da Norris, è possibile che comparazioni temporali offrano un modo per evitare che le distorsioni costituiscano un errore significativo. Tuttavia, la validità dei dati rilevati non può che dipendere da una attenta formulazione dei questionari volti ad individuare le opinioni dei cittadini - attenzione che spesso, data la necessità di ricorrere a dati secondari, non può che essere limitata.

3. Lo studio empirico della legittimità fra opinioni e comportamenti: il contributo di Bruce Gilley

Nella seconda sezione è stato sottolineato come lo studio empirico basato su di una concezione 'oggettivista' della legittimità, tipica degli studi a livello macro, vada a sovrapporsi con lo studio della 'democraticità' o della 'qualità della democrazia'. E' stata, in seguito, discussa la soluzione 'soggettivista' allo studio della legittimità, che, seguendo la classica definizione weberiana, individua nelle 'credenze' dei soggetti al

${ }^{31}$ D. EAston, A Re-Assessment of the Concept of Political Support, cit., p. 436.

32 P.H. Merke, Comparing Legitimacy and Values Among Advanced Democratic Countries, in M. Dogan (a cura), "Comparing Pluralist Democracies: Strains of Legitimacy", Boulder, Westview Press, 1988, p. 23. 
potere il locus della legittimità. Tuttavia, sono stati evidenziati alcuni problemi della ricerca empirica tipici di questo secondo filone di studi, non in relazione alla concezione di legittimità che ne costituisce la cornice teorica, bensì sulla base dei possibili problemi metodologici a cui le ricerche empiriche a livello micro sono facilmente soggette.

In questa sezione conclusiva si tenterà di offrire un possibile spunto di riflessione metodologica sullo studio empirico della legittimità, a partire dai lavori di Bruce Gilley ${ }^{33}$. Se la legittimità è definita empiricamente come la proprietà di un sistema politico nel quale gli atteggiamenti dei governati sono orientati favorevolmente all'accettazione di qualsiasi decisione prodotta, a prescindere dal contenuto della decisione stessa, allora investigare empiricamente la legittimità comporta investigare gli atteggiamenti della comunità politica verso il sistema politico. I dati ricavati da opinion surveys, se ben analizzati, restano quindi il metodo principale di investigazione della legittimità. Tuttavia, non sembrano dover essere l'unico. Un possibile metodo di controllo per valutare l'affidabilità dei dati ottenuti sulla base di surveys, è considerare dati empirici relativi a comportamenti rilevanti. Ovviamente, il rapporto fra atteggiamenti e comportamenti è uno di causa e effetto ${ }^{34}$. Investigare i comportamenti dei cittadini potrebbe, quindi, offrire una misura indiretta dei loro atteggiamenti nei confronti del sistema politico. Il modello di studio empirico della legittimità in settantadue paesi proposto da Gilley sembra rappresentare un valido tentativo, da un lato, di raffinare i criteri di valutazione dei dati di surveys, dall'altro, di includere l'analisi di comportamenti rilevanti nello studio empirico della legittimità.

Lo studio di Gilley, offre, in primo luogo, la possibilità di rivedere la bipartizione fra studi a livello macro e studi e livello micro. Gilley, infatti, identifica una netta distinzione fra le cause della legittimità e l'esistenza della stessa. Lo studio delle caratteristiche proprie di un sistema politico non è, come per gli studi macro, un metodo per verificarne la legittimità. È invece, secondo Gilley, un modo per identificare quali fattori (che lui distingue fra politici e socio-economici) abbiano un impatto sulla legittimità di un sistema politico. La legittimità, in-

33 B. Gilley, The Meaning and Measure of State Legitimacy, cit.; B. GILlEy, The Determinants of State Legitimacy, cit..

${ }^{34}$ D. EAston, A Re-Assessment of the Concept of Political Support, cit., p. 436. 
vece, è da investigare empiricamente a livello micro, focalizzandosi non su 'fattori oggettivi', bensì sugli atteggiamenti dei cittadini stessi. Solo distinguendo fra la misura della legittimità e le sue cause è possibile offrire una chiara visione della legittimità di un sistema politico, nonché stabilire correlazioni di un certo rilievo empirico (ad esempio, con un indice di stabilità politica).

In questa sede mi concentrerò sulla presentazione della proposta di Gilley di misurazione della legittimità, tralasciando, quindi, la sua proposta di spiegazione delle cause. Al fine di proporre un metodo di investigazione empirica della legittimità, Gilley scompone il concetto in tre distinte dimensioni ${ }^{35}$. Nella definizione delle dimensioni della legittimità, Gilley si rifà alla tripartizione proposta da Beetham ${ }^{36}$, qui introdotta nella prima sezione. Tuttavia, a differenza di Beetham, Gilley non considera le tre dimensioni quali condizioni 'oggettive' di legittimità che, in quanto proprietà intrinseche di un sistema politico, possano essere rilevate da parte di un osservatore esterno. Per Gilley, il soddisfacimento delle varie condizioni può essere verificato empiricamente solo sulla base delle opinioni e dei comportamenti dei membri del sistema politico.

Per investigare, quindi, il grado in cui un sistema politico è 'creduto' legittimo dai propri cittadini, l'analisi si deve concentrare sulla presenza di quelle che Gilley chiama le visioni della legalità, le visioni della giustificazione e gli atti di consenso ${ }^{37}$.

1. La legalità riguarda la presenza di un accordo fra il modo in cui il potere politico è acquisito e viene esercitato, e le visioni dei cittadini riguardo alla legge.

2. La giustificazione riguarda la presenza di un accordo fra le ragioni morali del possesso e dell'esercizio del potere e i principi, le idee e i valori condivisi dai cittadini.

3. Gli atti di consenso, come è facile intuire, si riferiscono a veri e propri comportamenti dei cittadini che esprimono un riconoscimento del diritto dei governanti ad essere obbediti 38 .

${ }^{35}$ B. GILley, The Meaning and Measure of State Legitimacy, cit..

36 D. Beetham, The Legitimation of Power, cit..

${ }^{37}$ B. GILLEY, The Meaning and Measure of State Legitimacy, cit., pp. 502-503.

${ }^{38} \mathrm{Si}$ veda anche R. Hardin, Compliance, Consent and Legitimacy, in C. Borx, S.C. Stokes (a cura), "Oxford Handbook of Comparative Politics", Oxford, Oxford University Press, 2007, pp. 236-255. 
Gilley riporta, quindi, la tripartizione sul piano della ricerca empirica, e considera le tre dimensioni sopra elencate come variabili costitutive del concetto di legittimità. La misura della legittimità di un dato sistema politico sarà quindi data dall'aggregazione dei dati ottenuti sulla base delle tre dimensioni. Gilley seleziona nove indicatori quantitativi, distribuiti per le varie dimensioni:

1. per la dimensione visioni della legalità si rifà a tre domande contenute nel World Values Survey (1999-2002) concernenti: 1) il rispetto dei diritti umani; 2) la fiducia nella polizia; 3) la fiducia nell'amministrazione pubblica. Esclude quindi, di proposito, dati concernenti il governo, parlamenti e partiti, poiché rischierebbero di registrare le opinioni verso "particolari governi piuttosto che lo stato stesso ${ }^{39}$ ". Opinioni verso il sistema giudiziario, sono, invece, escluse per mancanza di dati;

2. per misurare, invece, la dimensione visioni della giustificazione, utilizza due tipi di indicatori:

- uno basato sulle opinioni, ovvero i dati sulle risposte a tre domande concernenti le opinioni riguardo al sistema politico nel suo complesso. E' da notare che il tipo di domande selezionato è quello cosiddetto 'realista', in quanto formulato appositamente per evitare valutazioni comparative rispetto ad un tipo 'ideale' di sistema politico, e permette, quindi, di cogliere le opinioni sull'attuale sistema politico 'così com'è', in riferimento soltanto ai cosiddetti 'valori condivisi' 40 . La maggior validità di misure 'realiste' della legittimità, in particolare per paesi di recente democratizzazione o dove la democrazia non è del tutto consolidata, è stata rilevata da Mishler e Rose ${ }^{41}$;

- uno basato sui comportamenti, nello specifico, la misura dell'incidenza della violenza nelle proteste politiche, sulla base di dati contenuti nel World Handbook of Political and Social Indicators $I V$. La scelta dell'incidenza della violenza nelle proteste politiche, e non, ad esempio, del numero totale di manifestazioni di protesta

${ }^{39}$ B. GiLLEy, The Meaning and Measure of State Legitimacy, cit., p. 507.

${ }^{40}$ B. GILLEY, The Meaning and Measure of State Legitimacy, cit., p. 507.

41 W. Mishler, R. Rose, Political Support for Incomplete Democracies: Realist Vs. Idealist Theories and Measures, in "International Political Science Review", n. 4, 2001, pp. 303-320. 
politica, è spiegata sulla base di due ragioni. La prima è che il numero e la frequenza di proteste in un particolare sistema politico sono, per lo meno in parte, intrinsecamente legate alla cultura politica di un determinato paese e, in questo senso, non utili al fine di una misura della legitttimità di un dato sistema. La seconda ragione è che l'uso della violenza, proprio per il suo carattere di eccezionalità, sembra essere un indicatore affidabile di opposizione al regime ${ }^{42}$;

3. per accertare, infine, la misura di atti di consenso, sceglie di concentrarsi su:

- dati empirici sul pagamento di tasse cosiddette 'quasi-volontarie', al fine di valutare il consenso verso la struttura socio-economica, sulla base di dati del Government Finance Yearbook (1996-2002) del Fondo Monetario Internazionale;

- dati empirici sulle elezioni nazionali (percentuale di votanti rispetto ai titolari di diritto di voto - e non rispetto ai registrati nell'ultima elezione politica) sulla base di dati forniti dall'International Institute for Democracy and Electoral Assistance IDEA.

$\mathrm{Al}$ fine di formulare, infine, una misura di legittimità, Gilley propone una media pesata $(25 \%, 50 \%$ e $25 \%)$ dei dati rilevati sulle varie dimensioni, considerando la seconda dimensione, ovvero quella riferita alle 'visioni della giustificazione', come più rilevante delle altre ai fini della misurazione della legittimità di un dato sistema politico. La buona correlazione dell'indice di legittimità di Gilley coi dati riguardanti la stabilità politica dei paesi inclusi nel suo studio sembra suggerire che l'inclusione di comportamenti, e non solo di opinioni, nella misurazione della legittimità possa essere considerata una scelta valida, e aprire il campo ad una promettente linea di ricerca.

${ }^{42}$ E.N. Muller, T.O. Jukam, M.A. Seligson, Diffuse Political Support and Anti-system Political Behavior: A Comparative Analysis, in "American Journal of Political Science", n. 2, 1982, pp. 240-265. 
Abstract - The concept of legitimacy is unquestionably one of the most diffused and yet ambiguous of the political language. To attribute legitimacy to a political object - political power, a political institution, a political system - means conferring to the same object some sort of positive valuation. Nonetheless, amongst scholars of legitimacy, agreement is still to be found about which property, or set of properties, a certain object should possess to be called legitimate. After introducing a formal analysis of the concept of legitimacy, this work proceeds by reviewing some of the major contemporary contributions to the definition, as well as the empirical measurement, of the legitimacy of a political system. In particular, this analysis distinguishes between definitions of legitimacy at the 'macro' level, that understand and measure legitimacy as given by specific characteristics of political institutions, and definitions at the 'micro' level, that investigate the legitimacy of a political system on the basis of the study of citizens' opinion and their support to the government. The concluding section introduces and briefly discusses Bruce Gilley's approach to legitimacy. While placing itself among the studies at the 'micro' level, Gilley's comparative study of legitimacy is able to overcome some of their theoretical and methodological problems. 